\title{
Loss of Snord116 impacts lateral hypothalamus, sleep, and food-related behaviors
}

\author{
Marta Pace, ${ }^{1}$ Matteo Falappa, ${ }^{1,2}$ Andrea Freschi, ${ }^{1}$ Edoardo Balzani, ${ }^{1}$ Chiara Berteotti, ${ }^{3}$ \\ Viviana Lo Martire, ${ }^{3}$ Fatemeh Kaveh, ${ }^{4}$ Eivind Hovig, ${ }^{4,5}$ Giovanna Zoccoli, ${ }^{3}$ Roberto Amici, ${ }^{6}$ \\ Matteo Cerri, ${ }^{6}$ Alfonso Urbanucci, ${ }^{4}$ and Valter Tucci ${ }^{1}$ \\ ${ }^{1}$ Cenetics and Epigenetics of Behaviour Laboratory, Istituto Italiano di Tecnologia, via Morego 30, Italy. ${ }^{2}$ Dipartimento \\ di Neuroscienze, Riabilitazione, Oftalmologia, Genetica e Scienze Materno-Infantili (DINOGMI), Università degli Studi \\ di Genova, Genova, Italy. ${ }^{3}$ PRISM Lab, Department of Biomedical and Neuromotor Sciences, Alma Mater Studiorum, \\ University of Bologna, Bologna, Italy. ${ }^{4}$ Department of Tumor Biology, Institute for Cancer Research, Oslo University \\ Hospital, Oslo, Norway. ${ }^{5}$ Centre for Bioinformatics, Department of Informatics, University of Oslo, Oslo, Norway. \\ ${ }^{6}$ Department of Biomedical and NeuroMotor Sciences, Alma Mater Studiorum, University of Bologna, Bologna, Italy.
}

Imprinted genes are highly expressed in the hypothalamus; however, whether specific imprinted genes affect hypothalamic neuromodulators and their functions is unknown. It has been suggested that Prader-Willi syndrome (PWS), a neurodevelopmental disorder caused by lack of paternal expression at chromosome 15q11-q13, is characterized by hypothalamic insufficiency. Here, we investigate the role of the paternally expressed Snord116 gene within the context of sleep and metabolic abnormalities of PWS, and we report a significant role of this imprinted gene in the function and organization of the 2 main neuromodulatory systems of the lateral hypothalamus (LH) - namely, the orexin (OX) and melanin concentrating hormone (MCH) - systems. We observed that the dynamics between neuronal discharge in the LH and the sleep-wake states of mice with paternal deletion of Snord116 (PWScr ${ }^{\mathrm{m}+/ \mathrm{p}-}$ ) are compromised. This abnormal state-dependent neuronal activity is paralleled by a significant reduction in OX neurons in the LH of mutant mice. Therefore, we propose that an imbalance between OX- and $\mathrm{MCH}$-expressing neurons in the $\mathrm{LH}$ of mutant mice reflects a series of deficits manifested in the PWS, such as dysregulation of rapid eye movement (REM) sleep, food intake, and temperature control.

Authorship note: MP and MF contributed equally to this work.

Conflict of interest: The authors have declared that no conflict of interest exists.

Copyright: (c) 2020, American Society for Clinical Investigation.

Submitted: February 28, 2020

Accepted: April 24, 2020

Published: June 18, 2020

Reference information: JCI Insight. 2020;5(12):e137495.

https://doi.org/10.1172/jci.

insight.137495.

\section{Introduction}

Both maternally and paternally derived genes are essential for survival beyond fertilization; these genes differentially affect embryonic brain development and, consequently, postnatal and adult physiology. In particular, paternally derived genes are thought to control the organization of the subcortical limbic system (1). For example, androgenetic ( 2 paternal copies) cells are mainly distributed in the hypothalamus, although the specific impact of such parental genetic information on hypothalamic functions remains unknown.

The hypothalamus is an ancient structure that orchestrates primitive physiological processes for survival (2), such as motivated behaviors for feeding and drinking, the regulation of body temperature, and the switch between sleep and wakefulness. Therefore, a number of paternally expressed genes and overlapping circuitries in the hypothalamus function as potential regulators of mammalian sleep and sleep-mediated metabolism (3). To this end, over the last decade, we have demonstrated that parent-of-origin imprinted genes exert a pivotal role in the control of sleep physiology and feeding behavior $(3,4)$

Among the pathological conditions that depend on genomic imprinting defects, Prader-Willi syndrome (PWS) is the neurodevelopmental disorder that, up to now, best describes the link between sleep, metabolism, and imprinted genes. PWS results from the loss of a cluster of paternally expressed genes on the chromosome 15q11-q13 region, many of which are highly expressed in the hypothalamus and are characterized by sleep-wake (rapid eye movement [REM] alterations) and metabolic (hyperphagia) abnormalities. All of these symptoms are generally associated with hypothalamic insufficiency (5-7). 
We have previously described that microdeletion of the small nuclear RNA 116 (SNORD116) cluster within the PWS locus induces REM and temperature dysregulations in mice and human subjects (5). Specifically, the deletion of Snord116 in mice causes an EEG profile characterized by the intrusion of REM sleep episodes into the transition between wakefulness and sleep accompanied by an increase in body temperature. REM sleep intrusions have been reported in several clinical studies in which PWS subjects manifest narcolepsy and express symptoms such as sleep attacks during active wakefulness, cataplexy (a transient loss of muscle tone during wakefulness), sleep paralysis, and sleep fragmentation (8). Narcolepsy is a sleep condition that causes the loss of hypothalamic OX neurons (also known as hypocretin; HCRT) (9), and previous studies have observed that subjects with PWS show OX alterations (10-12).

OX neurons are located in the lateral hypothalamus $(\mathrm{LH})$, where this class of neurons promotes wakefulness (13) by facilitating the release of other arousal-promoting brain neuromodulators (noradrenaline, histamine, and acetylcholine) (14). However, in the LH, OX neurons are intermingled with a group of neurons that release the melanin-concentrating hormone $(\mathrm{MCH})$ and promote sleep, and these neurons are active mainly during REM sleep (15). Both OX and MCH neurons project widely throughout the brain, exerting antagonistic actions on brain states and energy balance. However, whether these 2 groups of neurons of the LH exert abnormal control over sleep-wake cycles, feeding and temperature in the PWS remains unclear.

In this study, we tested the hypothesis that the loss of paternally expressed Snord116 disrupts specific neuromodulatory systems of $\mathrm{LH}$, particularly those that overlap in the control of sleep, feeding, and temperature. We found that mice with paternal deletion of Snord116 have altered dynamics in how neuronal activity of the LH is associated with sleep-wake cortical states and feeding. This altered modulation between cortical states, behavior, and subcortical neuronal activity in Snord116-deleted mice is accompanied by a loss of OX-expressing neurons in the LH. We also report, for the first time to our knowledge, a link between Snord116 and a different paternally imprinted gene, Peg3, which has been recently associated with the control of the hypothalamic OX neuromodulatory system (16).

\section{Results}

Loss of paternal Snord116 alters neuronal dynamics in the LH associated with sleep homeostasis. To investigate whether the firing pattern of neurons within the LH manifests signs of paternal-dependent subcortical regulation throughout different arousal states of the brain, we studied mice with paternal deletion of the Snord116 gene (17), PWScr ${ }^{\mathrm{m}+\mathrm{p}-}$ mice, and their WT littermate controls, $\mathrm{PWScr}^{\mathrm{m}+\mathrm{p}^{+}}$mice. A total of 569 well-sorted units were successfully recorded from $\mathrm{PWScr}^{\mathrm{m}+/ \mathrm{p}+} \mathrm{WT}$ mice, and 548 units were recorded from $\mathrm{PWScr}^{\mathrm{m}+/ \mathrm{p}-}$ mutant mice across all time points. Based on the distribution of the firing activity of each unit, we were able to distinguish populations of putative neurons in the LH that were time-locked with the occurrence of specific sleep-wake states (Figure 1, C and E). In particular, we identified neurons (Figure 1E) associated with sleep (S-max) or wakefulness (W-max). However, we distinguished between non-REM (NREM) (NR-max), REM (R-max) neurons and neurons firing in both stages (NRR-max) (Supplemental Table 1; supplemental material available online with this article; https://doi.org/10.1172/ jci.insight.137495DS1). We also identified a group of neurons that did not show significant changes in their firing rate across the sleep-wake states, and we called this latter class of neurons ws (wake and sleep).

Next, we identified LH neurons that are time-locked to food intake. In this experiment, a total of 147 units were identified from $\mathrm{PWScr}^{\mathrm{m}+/ \mathrm{p}+}$ mice and 74 units were identified from the $\mathrm{PWScr}^{\mathrm{m}+/ \mathrm{p}-}$ mice in response to food deprivation (FD). Neurons were classified as Type I (fire maximally after nose-poking/feeding activity), Type II (fire maximally before nose-poking/feeding activity), and Type III neurons (not-responding neurons) (Figure 1, D and F).

Strikingly, we observed a significant increase in S-max and a reduction in W-max neurons in $\mathrm{PWScr}^{\mathrm{m}+/ \mathrm{p}-}$ mutant mice compared with controls at baseline (BL) (Figure 1G and Supplemental Table 2). A more detailed analysis of the BL (BL 1 [B1] and B2, as shown in Figure 1G) confirmed this distribution of the classes of LH neurons according to EEG states (Supplemental Figure 1B and Supplemental Table 2). Moreover, when the pressure of sleep is low (B1), mutant mice exhibit more REM sleep than their littermate controls (Supplemental Figure 1A). This finding suggests that the pressure/ need of sleep may be either a permissive or a blocking mechanism with respect to excess REM sleep due to the lack of Snord116. This phenomenon is confirmed by the profile of the power densities in REM sleep (Supplemental Figure 1C). Indeed, in $\mathrm{PWScr}^{\mathrm{m}+/ \mathrm{p}-}$ mutant mice, the theta $(4.5-9 \mathrm{~Hz}$ ) band, which 
A

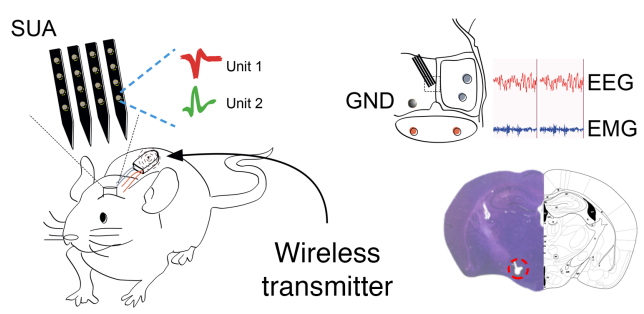

B

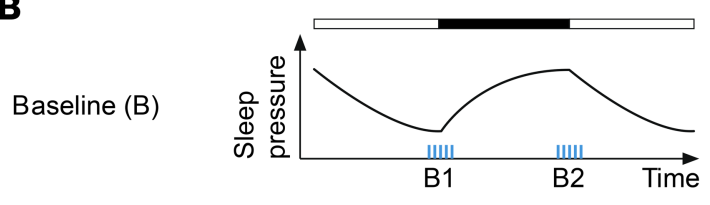

Sleep Deprivation (SD)
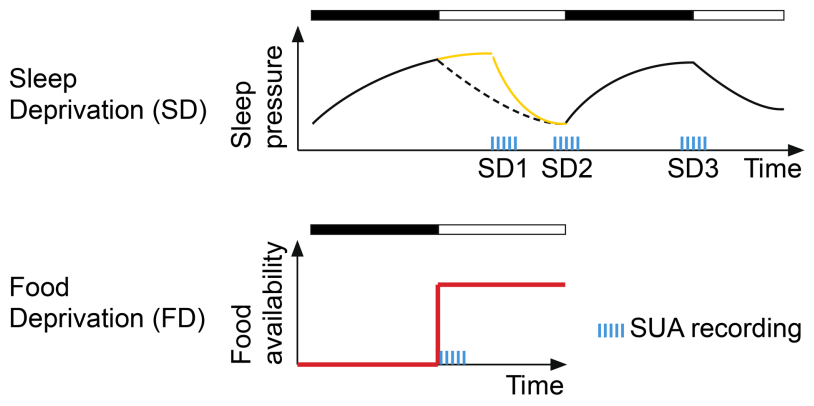

C EEG MAlw

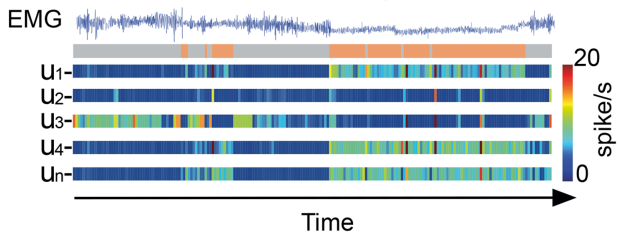

D

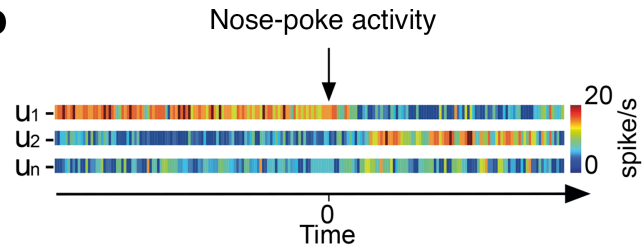

E

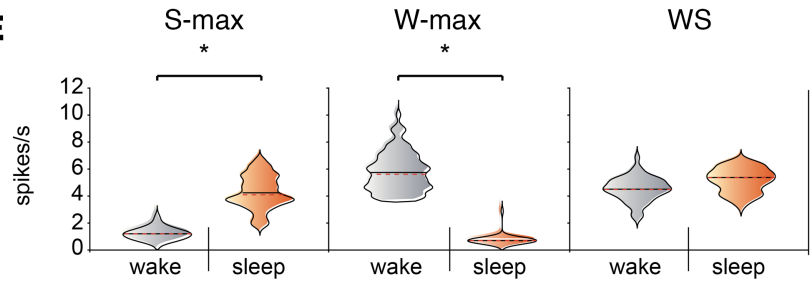

$\mathbf{F}$

G
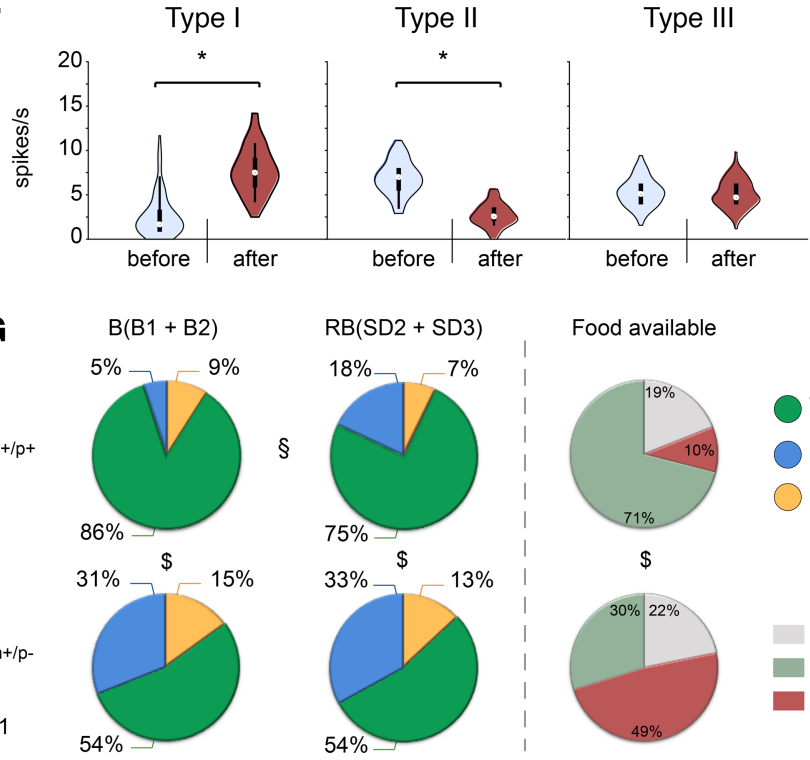

Food available

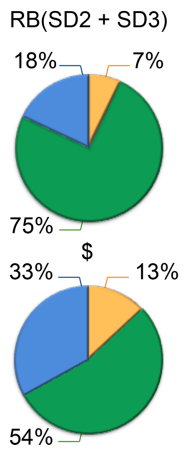

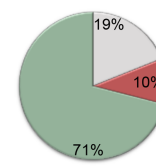

W-max

S-max ws

$\$$

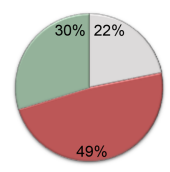

Figure 1. Loss of paternal Snord116 alters neuronal dynamics in the LH associated with sleep and food. (A) The cartoon shows mice chronically implanted with a microwire array of 16 channels and with an EEG-EMG wireless transmitter. The correct placement of the SUA electrode was histologically verified by $40-\mu \mathrm{m}$ Nissl-stained coronal brain sections (bregma, -1.10/-1.90). (B) The experimental design used to record SUA and the sleep-wake cycle. (C) An example of sleep stages (wakefulness in gray; sleep, including both NREM and REM sleep stages, in orange) aligned with the firing rate recorded in the LH. The heatmaps show the response firing rate in spikes/second from $0 \mathrm{~Hz}$ (blue) to $20 \mathrm{~Hz}$ (red). (D) The heatmaps show the response firing rate used to classify neurons before and after food consumption (firing rate in spikes/second from $0 \mathrm{~Hz}$ [blue] to $20 \mathrm{~Hz}$ [red]). (E) Violin plots of classified units according to the sleep-wake cycle according to ANOVA followed by post hoc Bonferroni's correction $(P<0.05)$. (F) Violin plots of classified units according to their discharge related to food consumption (paired Student's $t$ test of the firing rate between before and after the pellet was released, binned at $50 \mathrm{~ms}, P<0.05)$. (G) The pie chart represents the distribution of recorded neurons according to the sleep-wake stage: wake (W-max, in green), sleep (both NREM and REM sleep, S-max, in blue), and not responding (ws, yellow). Food-related neurons are classified as Type I neurons, in gray; Type II neurons are in green; and Type III neurons are in red. Differences between the 2 genotypes are indicated by $\$$, while differences within groups across time points are indicated by $\S$. Significance was computed with the $\chi^{2}$ test; for details on the statistical analysis, see Supplemental Tables 2-4. The 2 genotypes investigated were PWScr ${ }^{\mathrm{m}+/ \mathrm{p}^{-}}$mice $(n=4)$ and PWScr ${ }^{\mathrm{m}+p^{\mathrm{p}+}}$ mice $(n=4)$.

is characteristic of REM sleep, is higher in both B1 and B2, but at B2, the EEG frequency phenotype is not accompanied by longer REM sleep duration, implying that the overall higher sleep pressure masks differences in microstructural aspects of electrophysiological sleep. Moreover, the delta $(0.5-4.5$ $\mathrm{Hz}$ ) band presents an opposite trend in the 2 groups of animals; counterintuitively, reduced power is observed in mutant mice as the pressure of sleep increases (Supplemental Figure 1C). Again, this phenomenon suggests abnormal homeostatic control of sleep in mutant mice.

Thus, we sought to explore the physiological responses of mice following 6 hours of total sleep deprivation (SD) by investigating the distributions of the neuronal classes at different time points during 18 hours of recovery (SD1, SD2, and SD3; Figure 1B). We observed that SD induced an increase in S-max neurons in WT PWScr ${ }^{\mathrm{m}+\mathrm{p}^{+}}$mice, while in mutant $\mathrm{PWScr}^{\mathrm{m}+\mathrm{p}-}$ mice, the distribution of classes 
of neurons remained unaltered compared with $\mathrm{BL}$ (Figure $1 \mathrm{G}$ ). This result indicates that control mice homeostatically respond to SD by modulating LH neuronal dynamics, as previously reported in the literature (18), while mutant mice lack this hypothalamic modulatory process.

Across different conditions, neurons may continue responding to the same state, may respond to a different state, or may become aspecific in their activity. Within the rearrangements of neuronal modulation across different phases, an interesting observation in our study is represented by the behavior of the nonresponding neurons following SD (Supplemental Figure 1A and Supplemental Table 3). In WT mice after $\mathrm{SD}$, the number of neurons of this latter class increases 3 times compared with the previous BL, a phenomenon that is gradually recovered over the following 18 hours after deprivation. This change comes with a cost for wake-dependent neurons in WT animals. In $\mathrm{PWScr}^{\mathrm{m}+/ \mathrm{p}-}$ mutant mice, this SD effect is missing, reinforcing the observation of a lack of neuromodulation caused by the paternally derived genetic defect.

Finally, the dramatic drop in delta and theta power densities in $\mathrm{PWScr}^{\mathrm{m}+/ \mathrm{p}-}$ mutant mice compared with controls at SD2 (Supplemental Figure 1C) coincides with the permissive role of sleep pressure at this time of the day for the sleep defects in mutant mice.

The LH is made up of a heterogeneous group of cells that express various neuropeptides, such as $\mathrm{OX}$ and $\mathrm{MCH}$ neurons. To gain insight about the heterogeneous nature of our recording units, we plotted the mean firing rate versus the mean logarithm of the electromyogram (EMG) signals across 3 groups of putative neurons (W-max; S-max and ws neurons) of the whole sleep experiment (Supplemental Figure 1D). The graphical representation of these neurons within the $2 \mathrm{D}$ scatter plot enables us to distinguish between $\mathrm{MCH}$ putative and $\mathrm{OX}$ putative neurons. $\mathrm{MCH}$ putative neurons fire maximally during REM sleep and are thought to present a low EMG amplitude and a low average discharge rate of $1.1 \pm 0.26 \mathrm{~Hz}$, while OX putative neurons fire maximally during wake and are characterized by a high firing rate, $3.17 \pm 0.79 \mathrm{~Hz}$ (19). Based on these criteria, we assessed $\mathrm{MCH}$ putative and OX putative neurons between $\mathrm{PWScr}^{\mathrm{m}+\mathrm{p}+} \mathrm{WT}$ and $\mathrm{PWScr}^{\mathrm{m}+/ \mathrm{p}-}$ mutant mice. The percentage of recorded $\mathrm{MCH}$ putative neurons was unchanged between the 2 genotypes; although nonstatistically significant, a total of 211 OX putative neurons were identified in $\mathrm{PWScr}^{\mathrm{m}+/ \mathrm{p}^{-}}$mutant mice compared with $315 \mathrm{OX}$ putative neurons identified in control mice (Supplemental Figure 1D).

$A$ high proportion of neurons in the LH do not respond to food intake in PWScr ${ }^{m+p-}$ mutant mice. Mice underwent FD during the dark period/resting phase (Figure 1B) to increase their drive for food intake. When food was made available again, pellets were automatically provided in the home cages of each mouse (see Methods). The frequency of nose-poke activity to gain food was similar between the 2 genotypes (PWScr ${ }^{\mathrm{m}+\mathrm{p}-}, 107$ trials; $\mathrm{PWScr}^{\mathrm{m+} / \mathrm{p}+}, 116$ trials), indicating no obvious behavioral differences between the 2 genotypes, as previously described $(20,21)$.

As for the sleep experiment, our attention fell on the distribution of neuronal modulation between the 2 genotypes. We found that $\mathrm{PWScr}^{\mathrm{m}+/ \mathrm{p}-}$ mutant mice had less than half the number of Type II neurons, which have reduced firing during food intake, of control mice (Figure 1G). This reduction may be explained by the fact that mutant mice had twice as many nonresponding neurons as control mice. Indeed, Type I neurons were almost identical in the 2 groups (Supplemental Table 4). These results also suggest an abnormal distribution of OX neurons in the LH of mutant mice, as OX neurons drive food intake.

Sleep homeostasis is disrupted in PWS mutant mice. We explored the 24-hour EEG profile of sleep (see Methods) and the responses to sleep loss in a different cohort of animals.

The results confirmed our previous observations that sleep is altered in the PWS mutant mice (5). In particular, REM sleep is increased in $\mathrm{PWScr}^{\mathrm{m}+/ \mathrm{p}-}$ mutant mice compared with controls, showing a peak increase at Zeitgeber time (ZT) 20 and an overall increase in theta power (Figure 2A). These experiments were conducted at standard room temperature of $22^{\circ} \mathrm{C}$. Interestingly, the theta profile in mutant mice did not show circadian rhythm (Figure 2A); $\mathrm{PWSCr}^{\mathrm{m}+/ \mathrm{p}-}$ mutant mice had an increase in theta power during the transition between light and dark, when the pressure of sleep is low. Overall, REM sleep abnormalities remained a selective phenotype in these mutant mice, while the other EEG-determined arousal states were unchanged between the 2 genotypes (Supplemental Figure 2A).

Moreover, we tested the homeostatic EEG response following SD in PWScr ${ }^{\mathrm{m}+/ \mathrm{p}-}$ mutant mice compared with littermate controls. Compared with control mice, $\mathrm{PWScr}^{\mathrm{m}+/ \mathrm{p}-}$ mice showed reduced delta activity during NREM sleep rebound following SD (Figure 2B), although the total amount of NREM sleep was unaltered over the long-term recovery process (Supplemental Figure 2A). Notably, delta power is the best electrophysiological marker of sleep propensity, although the temporal distribution of its rebound 
following SD is less characterized. The behavior of delta sleep in mutant mice suggests a dysregulation in the daily distribution of sleep pressure in these mice.

Our results also show a significant decrease in REM sleep during the first 2 hours of rebound in mutant mice compared with controls (Figure 2A), confirming both the alteration of homeostatic control in mutant mice and the alteration of REM sleep.

Thermoneutrality does not increase REM sleep in mutant mice. REM sleep is an evolutionarily recent physiological state of sleep that, in mammals, is largely dependent on the environmental temperature $(22,23)$. The maximum REM sleep expression in mice is attained when the environmental temperature is near $29^{\circ} \mathrm{C}$. The latter represents a thermoneutral zone (TNZ) for this species (24). In a new set of experiments, we modified the temperature environment of the animals to allow the duration of REM sleep to reach a maximum (24). Mice were housed for 5 weeks at $30^{\circ} \mathrm{C}$; then, EEG/EMG were recorded for 24 hours at the TNZ

We observed that $\mathrm{PWScr}{ }^{\mathrm{m}+\mathrm{p}-}$ mice showed a significant increase in the distribution of sleep stages (total sleep, NREM, and REM sleep; Supplemental Figure 2B) at $30^{\circ} \mathrm{C}$ during the dark period at ZT 20; in mice, these increases account for high sleep pressure $(25,26)$. These data suggest that the TNZ facilitate the accumulation of sleep pressure during the active period (dark period) in the mutant mice (Figure $2 \mathrm{~A}$ and Supplemental Figure 2A). The NREM sleep delta power and the REM sleep theta power were unchanged between the 2 genotypes, (Supplemental Figure 2, C and D).

Peripheral thermoregulatory responses are absent in PWS mutant mice. We tested whether loss of paternally expressed Snord116 affects peripheral responses and whether these changes impact the overall body temperature and body weight of the animals. We monitored the peripheral and cutaneous body temperature at the head (T-head) and tail (T-tail), and consequently, we derived the heat loss index (HLI) (see Supplemental Methods). Recordings were made at room temperatures of both $22^{\circ} \mathrm{C}$ and $29^{\circ} \mathrm{C}-30^{\circ} \mathrm{C}$ (TNZ). The TNZ imposes body temperature adjustments by changing the vasomotor tone in specialized heat exchange organs, such as the tail, in mice (27). We observed an increase in the vasculature tail skin tone at the TNZ in both genotypes compared with $22^{\circ} \mathrm{C}$ (Figure $2 \mathrm{C}$, upper panel). However, greater tail vasodilatation was observed in control mice, particularly between the switch from light to dark periods. These results indicate that, while at TNZ, WT control mice present a proper peripheral thermoregulatory response when the pressure of sleep is low, which is instrumental in maintaining the core body temperature of the animal at this time of the day; mutant mice lack such an important physiological response. A significant increase in the HLI was recorded in both genotypes, but a greater increase was found in PWScr ${ }^{\mathrm{m}+\mathrm{p}+}$ WT mice than in mutant mice (Figure 2C, middle panel). We also observed that the peripheral body temperature recorded at $22^{\circ} \mathrm{C}$ increased in $\mathrm{PWScr}^{\mathrm{m}+/ \mathrm{p}-}$ mutant mice compared with control mice during the light period (Figure $2 \mathrm{C}$, bottom panel), suggesting a diminished homeostatic control when sleep is physiologically facilitated in mice. Moreover, mutant mice failed to show the circadian oscillatory profile of temperature at room temperature that is present in $\mathrm{PWScr}^{\mathrm{m}+\mathrm{p}+}$ mice. However, at TNZ, the difference between the 2 genotypes during the light period was no longer observed. Furthermore, in agreement with previous reports (21), we observed that $\mathrm{PWScr}^{\mathrm{m}+/ \mathrm{p}-}$ mutant mice had a smaller body size when kept either at $22^{\circ} \mathrm{C}$ and at $30^{\circ} \mathrm{C}$ later in their development (for only 5 weeks) and when they were exposed at $30^{\circ} \mathrm{C}$ from birth and kept at this temperature for up to 20 weeks of age (Supplemental Figure 3).

Lack of Snord116 impairs the OX system in the LH. To assess the regulation of the main neuropeptides in the $\mathrm{LH}$, we examined the expression of $\mathrm{OX}$ and $\mathrm{MCH}$. Thus, we assessed the precursor of $\mathrm{MCH}(P m c h)$ and the prepro-OX (Ppox). We examined 3 conditions (Supplemental Figure 4A); at ZT 6, we tested both the BL condition (T0) and the effects of SD (T1), and at ZT 7, 1 hour after SD (T2), we tested the effects during the recovery from the loss of sleep.

We report that $\mathrm{PWScr}^{\mathrm{m}+\mathrm{p}-}$ mutant mice showed a significant increase in Ppox at T0 BL compared with control mice, while Pmch was unchanged in both genotypes at T0 (Figure 2D). At T1, both Ppox and Pmch were significantly reduced in $\mathrm{PWScr}^{\mathrm{m}+/ \mathrm{p}-}$ mice compared with control mice (Supplemental Figure 4B), confirming that the homeostatic response in mutant mice is reduced and that these 2 systems are dysregulated in mutant mice. At T2, the difference between the 2 genotypes for Ppox and Pmch was repristinated (Supplemental Figure $4 \mathrm{~B})$, confirming that the effects of SD in mutant mice are only acute and follow the immediate response after SD.

Then, we evaluated the receptors for $\mathrm{MCH}$ and OX. We assessed mRNA levels of OX receptor-1 $(O x 1 R), O x 2 R$, and $\mathrm{MCH}$ receptor $1(M c h 1 R)$ in the hypothalamus and in other brain areas as control measures. The expression levels were overall unchanged between the 2 genotypes, except for $O x 2 R$ in the parietal cortex (Supplemental Figure 4C). 
A

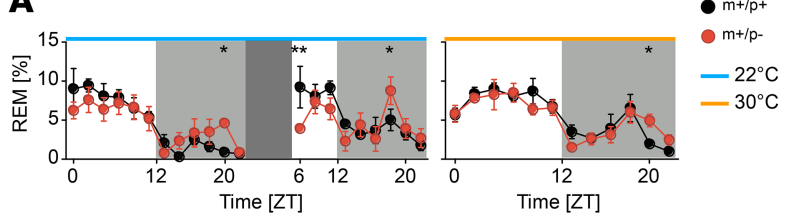

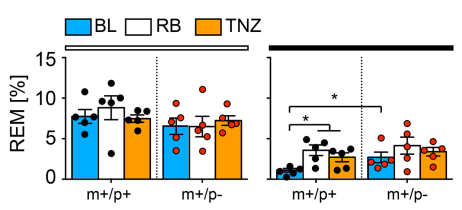

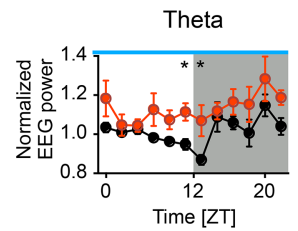

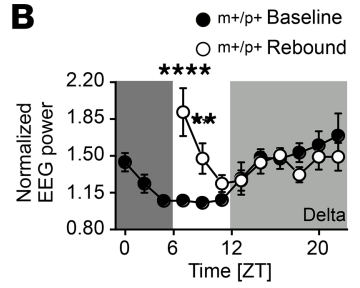
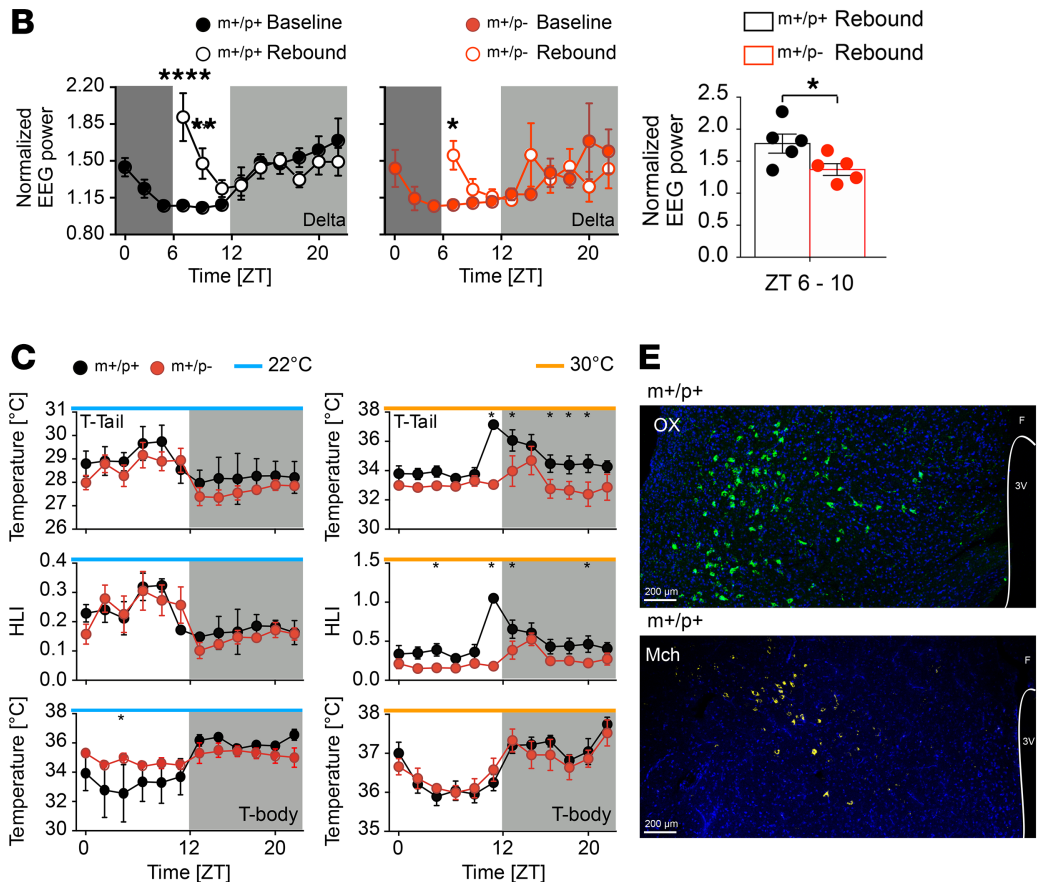

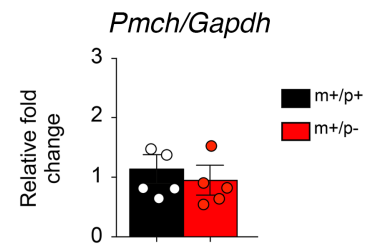

Figure 2. Homeostatic regulation and thermoregulatory response. (A) REM distribution in PWScr ${ }^{\mathrm{m}+/ \mathrm{p}^{+}}$and PWScr $\mathrm{Pn}^{\mathrm{m} / \mathrm{p}-}$ mice over a 24-hour (LD:12/12). Baseline (BL), 6 hours sleep deprivation (SD), and 18 hours rebound (RB) are shown. Data are shown as 2 -hour bin percentages. Conditions: $22^{\circ} \mathrm{C}$ and $30^{\circ} \mathrm{C}$ (TNZ). REM differences across the 2 cohorts (2-way ANOVA; time, $\mathrm{F}_{[20,160]}=8.66, \mathrm{P} \leq 0.0001$; genotypes, $\left.\mathrm{F}_{[8,160]}=3.33, \mathrm{P} \leq 0.001\right)$; at TNZ (2-way ANOVA; time, $\left.\mathrm{F}_{[11,88]}=11.61, \mathrm{P} \leq 0.0001\right)$. PWScr ${ }^{\mathrm{m}+/ \mathrm{p}-}$ mice showed an increase in REM sleep $\left(\mathrm{F}_{[1.85,4.74]}=18.06, P=0.0082\right)$. Theta power in REM increased in mutants (2-way ANOVA; $F_{[11,88]}=3.08, P \leq .001$ "time"; $F_{(1,8)}=8.04, P=0.02$ "genotypes"). (B) PWScr ${ }^{m+/ p+}$ mice displayed an increased delta from ZT6 to ZT10 (2-way ANOVA: $F_{[11,88]}=28.77 \mathrm{P} \leq 0.0001$ "interaction"), while mutants showed a mild increase at ZT 6 (2-way ANOVA; $F_{[11,88]}=12.25$ $P \leq 0.0001$ "interaction"). PWScr ${ }^{\mathrm{m}+/ \mathrm{p}-}$ mice showed lower delta than PWScr ${ }^{\mathrm{m}+/ \mathrm{p}+}$ mice in RB (unpaired $t$ test: $\left.\mathrm{t}_{[8]}=2.31, P=0.04\right)$. PWScr ${ }^{\mathrm{m}+/ \mathrm{p}^{-}}$mice $(n$ $=10,5$ mice at $22^{\circ} \mathrm{C}$ and 5 mice at $30^{\circ} \mathrm{C}$ ) and PWScr ${ }^{\mathrm{m+} / \mathrm{p}+}$ mice $\left(n=10,5\right.$ mice at $22^{\circ} \mathrm{C}$ and 5 mice at $\left.30^{\circ} \mathrm{C}\right)$. (C) T-tail, Heat loss index (HLI), and body temperature profiles are expressed as 2-hour mean \pm SEM. Mutants showed increased body temperature at $\mathrm{ZT} 6$ at $22^{\circ} \mathrm{C}\left(2-\mathrm{Way} \mathrm{ANOVA}\right.$ : $\mathrm{F}_{[11,88]}=$ 3.53, $P=0.0004$ "time"; $\mathrm{F}_{[11,88]}=7.86, P \leq 0.0001$ "genotypes"). At 30 C, T-tail (2-way ANOVA; $\mathrm{F}_{[11,88]}=2.68, P \leq 0.0001 ;$ "interaction") and HLI (2-way ANOVA; main effect of time of day, $\mathrm{F}_{[11,88]}=4.72, P \leq 0.0001$ "interaction") were increased in PWScr ${ }^{\mathrm{m}+\mathrm{p}+}$ mice. $\mathrm{PWScr} \mathrm{m}^{\mathrm{m} / \mathrm{p}-\mathrm{mice}}\left(\mathrm{n}=10,5 \mathrm{mice}\right.$ at $22^{\circ} \mathrm{C}$ and 5 mice at $30^{\circ} \mathrm{C}$ ) and PWScr ${ }^{\mathrm{m}+/ \mathrm{p}+}$ mice ( $n=10,5$ mice at $22^{\circ} \mathrm{C}$ and 5 mice at $30^{\circ} \mathrm{C}$ ). (D) PWScr ${ }^{\mathrm{m}+/ \mathrm{p}-}$ mice showed an increase in Ppox (unpaired $t$ test; $\mathrm{t}_{[8]}=2.49, P=0.03$ ). (E) Cell count distribution of OX immunoreactive neurons (upper) and MCH immunoreactive neurons (below) in the lateral hypothalamus. Coronal sections were stained with OX- and MCH-specific antibodies, counterstained with DAPI, and scored. PWScr ${ }^{\mathrm{m}+/ \mathrm{p}-}$ mice $(n=4)$ and PWScr ${ }^{\mathrm{m}+\mathrm{p}+}$ mice $(n=4)$. OX+ neurons were reduced in the PWScr ${ }^{\mathrm{m}+\mathrm{p}-}$ mutants (unpaired $t$ test; $\mathrm{t}_{[33]}=3.85, P=0.0005$ ). Values are expressed as the percentage of positive neurons relative to all stained nuclei (mean $\pm \mathrm{SEM}$ ). ${ }^{*} P \leq 0.05 ;{ }^{* *} P \leq 0.01 ;{ }^{* * *} P \leq 0.001 ;{ }^{* * * *} P \leq 0.0001$.

The overall contrast between neuropeptides and the mRNA levels of their receptors prompted us to quantify whether these chemical changes within the $\mathrm{OX}$ and $\mathrm{MCH}$ systems had consequences for the organization of the neuronal populations of the LH. We observed a significant reduction in OX neurons in the LH of $\mathrm{PWScr}^{\mathrm{m}+/ \mathrm{p}-}$ mutant mice (PWScr ${ }^{\mathrm{m}+/ \mathrm{p}+}$ mice $95 \pm 9 ; \mathrm{PWScr}^{\mathrm{m}+/ \mathrm{p}-}$ mice $53 \pm 10 ; P=0.0005$; Figure $2 \mathrm{E}$ ), while $\mathrm{MCH}$ neurons, which are located near the OX neurons, were unaffected $\left(\mathrm{PWScr}^{\mathrm{m}+/ \mathrm{p}+}\right.$ mice $66 \pm 5 ; \mathrm{PWScr}^{\mathrm{m}+/ \mathrm{p}-}$ mice $63 \pm 5$; Figure 2E). The discrepancy between the increased Ppox and the reduced neuronal population may be due to a mechanism to compensate for the low number of neurons with an overproduction of the peptide, as it has been already described in Magel2-KO mice, which accounts for another mice model of PWS (28).

Loss of Snord116 leads to transcriptional reprogramming in the hypothalamus. Next, we investigated whether the loss of Snord116 affects overall gene expression in the whole hypothalamus of $\mathrm{PWScr}^{\mathrm{m}+\mathrm{p}^{-}}$mutant mice compared with $\mathrm{PWSCr}^{\mathrm{m}+/ \mathrm{p}^{+}}$control mice by performing RNA sequencing (RNA-seq). The mice were investigated at the beginning of the light period ZT 0 (group 1; G1), 6 hours later at ZT 6 (G2), and 
at ZT 6 but following 6 hours of SD (G3). First, we sought to identify the differentially expressed genes (DEGs) between the 2 genotypes in G1. We identified 4777 downregulated genes and 4271 upregulated genes between genotypes (adjusted $P<0.05$; see Methods) and a minimum fold change of 2 (Supplemental Table 8). Next, we compared the down- and upregulated DEGs (Figure 3, A and B) in G1 PWScr ${ }^{\mathrm{m}+/ \mathrm{p}^{-}}$ mutant mice with DEGs derived from postmortem hypothalamic data from PWS patients versus healthy controls (29). Approximately 40\% of mouse DEGs overlapped with human DEGs, suggesting that loss of Snord116 recapitulates approximately $40 \%$ of the transcriptional changes found in the human hypothalamus (Figure 3, A and B; hypergeometric test, $P=5.308548 \times 10^{-12}$ ). To more closely evaluate the functional importance of these overlapping DEGs, we performed gene ontology (GO) enrichment analysis using Metascape (30). We found enrichment of several biological processes important for normal neural functions among the downregulated DEG, while inflammatory systems were the processes substantially enriched among the upregulated DEGs (Figure 3, A and B).

Interestingly, we found that Ppox was markedly downregulated in $\mathrm{PWScr}^{\mathrm{m}+/ \mathrm{p}^{-}}$mutant mice and in human PWS patients (Figure 3C), confirming that OX is involved in the abnormal regulation of REM sleep in PWS.

Next, we focused on how SD affects gene expression in the hypothalamus in both normal and $\mathrm{PWScr}^{\mathrm{m}+1}$ ${ }^{\mathrm{p}-}$ mutant mice. We compared DEGs in G2 versus G3 of each genotype (Figure 3D). We found that 6 hours of SD largely influenced the transcriptome of mutant mice (833 DEGs) compared with control mice (30 DEGs). Surprisingly, in the mutant data set, most of the DEGs were downregulated (804 genes downregulated versus 29 upregulated). GO analysis of all 833 DEGs revealed enrichment of cell organization, development, and growth-related processes (Figure $3 \mathrm{E}$ ), indicating that poor sleep negatively impacts the hypothalamus in PWS subjects, suggesting a clinical implication of these results. Finally, we investigated the RNA-seq data set to determine whether Snord116 loss significantly affects the expression of imprinted genes in humans and mice (Supplemental Table 9). Maternally expressed imprinted genes, such as $\mathrm{Meg} 3$, Gnas, and H19, as well as paternally expressed imprinted genes, such as Nap 115 and Peg3, were differentially expressed in mutant mice before and after SD (Figure 3F).

OX regulation in the hypothalamus depends on 2 paternally expressed genes, Snord116 and Peg3. We next sought to confirm selected physiologically relevant DEGs identified within the RNA-seq data set by quantitative PCR (qPCR). Among maternally and paternally imprinted genes that were altered in the hypothalamus of $\mathrm{PWScr}^{\mathrm{m}+\mathrm{p}-}$ mutant mice, we confirmed that $P e g 3$ showed a remarkable increase in expression in PWScr ${ }^{\mathrm{m}+/ \mathrm{p}-}$ mice (Figure 4A) compared with the control mice (Supplemental Figure 5A). PEG3 binds to DNA based on its multiple zinc finger motifs and nuclear localization $(31,32)$. Interestingly, a recent study found an association between Peg3 and OX expression (16). However, whether PEG3 is able to bind and regulate the expression of $\mathrm{OX}$ in living mice remains unclear. We noticed that 2 conserved promoter regions within a 3.2-kb fragment located upstream of the Ppox gene (Supplemental Figure 5F) target specific expression within the LH (33). We showed that PEG3 binds one of these regions. Specifically, ChIP (34) followed by qPCR (ChIP qPCR; Figure 4A) revealed that PEG3 binds the $2.5 \mathrm{~kb}$ OX regulatory element region in the hypothalamic mouse brain. Interestingly, a significant reduction in PEG3 binding was observed in $\mathrm{PWScr}^{\mathrm{m}+/ \mathrm{p}-}$ mice (Figure 4A), which may be associated with a significant reduction in OX-expressing neurons in mutant mice. Our results show that PEG3 positively regulates the expression of $P$ pox by enhancing its expression, as indicated by the presence of a strong enrichment of the H3K4me2/3 in the same region (Figure 4A). Conversely, H3K27me3, which is a marker of condensed chromatin (35), showed no enrichment in either genotype. The Gapdh promoter was used as a negative control for the ChIP experiment (Supplemental Figure 5E). These data reinforce the evidence that PEG3 contributes to the regulation of Ppox by enhancing its expression (Figure 4A).

Then, we sought to investigate whether Peg3 or Snord116 plays any causal role in the regulation and formation of OX neurons, and we assessed the expression of these 2 genes in OX-deficient mice. Specifically, we used 2 genetic mouse models of OX deficiency, Ppox-KO mice (9) and OX neuron-ablated (ataxin-3; Atx) mice (36) (Figure 4B).

Atx mice selectively degenerate postnatally, and a loss of $99 \%$ of neurons or more occurs by the age of 4 months (36). Therefore, in Atx mice, not only are OX neurons ablated, but cotransmitters are also eliminated (37). Conversely, KO mice are only deficient in Ppox (Figure 4B).

Interestingly, we observed that Peg3 was significantly increased in Atx mice compared with KO mice $(P=0.03)$. Moreover, Snord116 was also increased in Atx mice compared with KO mice and control mice $(P=0.03$; Figure 4B). Other imprinted genes tested were unchanged between genotypes (Supplemental 
A

Down regulated genes $\begin{array}{ll}\text { Humans } & 704\end{array}$

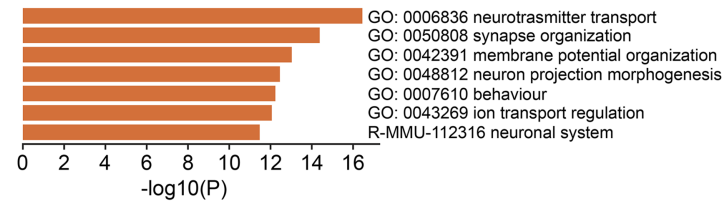

B

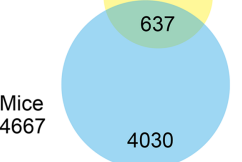

Up regulated genes
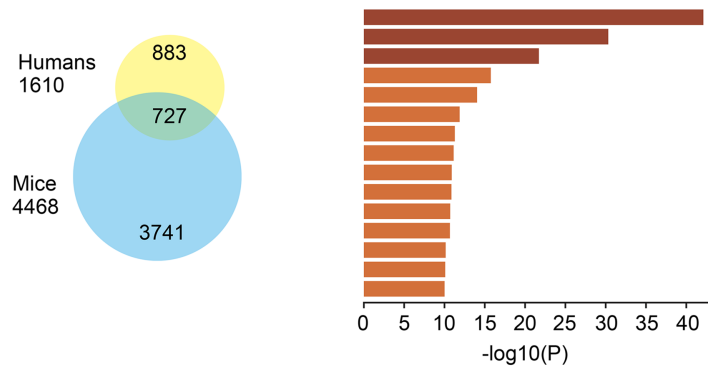

R-MMU-975956NMD independent of the EJC GO: 0006954 inflammatory response GO: 00019164 vasculature development GO: 0097190 apoptotic signaling GO: 0019221 cytokine-medieted signaling GO: 0002237 response to bacterial origin molecule GO: 0008630 intrinsic apoptotic signaling GO: 0008285 negative regulation of cell proliferation GO: 0007169 transmembrane receptor protein GO: 0002521 leukocyte differentiation mmu04657 IL-17 signaling . 0043086 negative regulation of catalytic activity GO: 0030099 myeloid cell differentiation

D

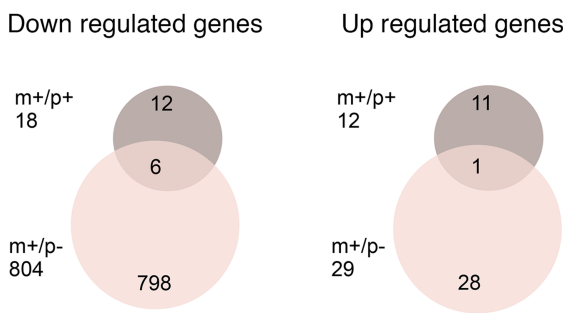

E

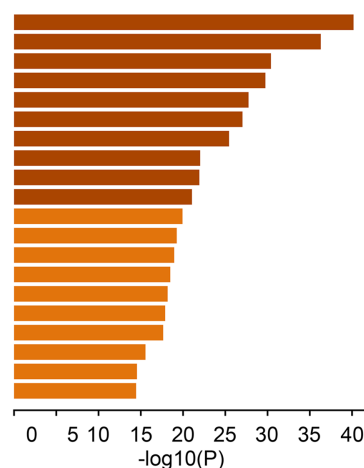

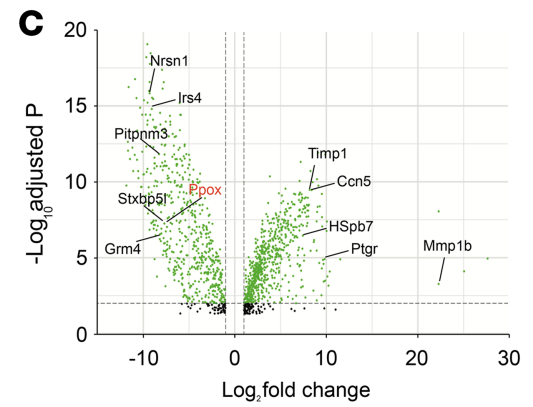

F $\quad \underline{G} \quad \underline{G} \quad \underline{G}$

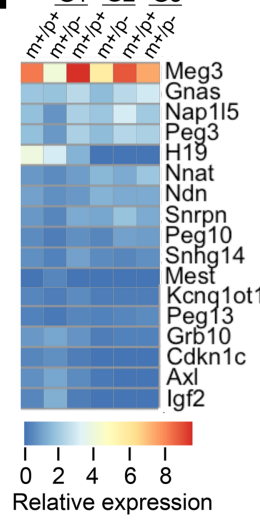

Figure 3. Snord116 loss significantly impacts molecular machinery in the hypothalamus. (A and B) Venn diagrams illustrating the number of differentially expressed genes (DEGs) that are downregulated (A) and upregulated (B) in the hypothalamus of Prader-Willi syndrome (PWS) PWScr ${ }^{\mathrm{m} / \mathrm{p}-}$ mice relative to control mice and that overlap in human patients (29). The results of gene ontology (GO) enrichment analysis of biological processes for the overlapping DEGs are also shown in both $\mathbf{A}$ and B. (C) Volcano plots of 637 and 727 DEGs in PWScr ${ }^{\mathrm{m}+/ \mathrm{P}^{-}}$mice in group 1 (G1; non-sleep deprived). (D) Significantly down- and upregulated genes in the hypothalamus of PWScr ${ }^{\mathrm{m+} / \mathrm{p}-}$ mutant mice compared with PWScr ${ }^{\mathrm{m}+/ \mathrm{p}+}$ control mice affected by sleep deprivation (G2 versus $\mathrm{C} 3$ ).

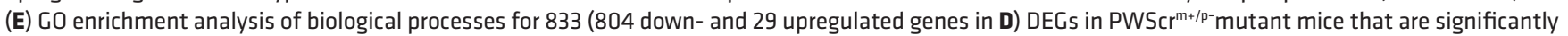
affected by sleep deprivation. (F) Heatmap of the relative expression of imprinted genes common in humans and mice assessed in PWScr ${ }^{\mathrm{m}+/ \mathrm{p}-}$ mutant mice compared with the PWScr ${ }^{\mathrm{m+} / \mathrm{p}+}$ mice in $\mathrm{G1}, \mathrm{G} 2$, and G3 (Supplemental Tables 8 and 9). PWScr $\mathrm{m}^{\mathrm{m} / \mathrm{p}-}$ mice and PWScrm+/p+ mice at 3 different time points at ZT 0 (G1; PWS, $n=3$, and WT, $n=3$ ), immediately after 6 hours of total SD (G2; PWS, $n=4$, and WT, $n=4$ ), and 1 hour after previous SD (C3, $n=4$; WT, $n=4$ ).

Figure 5B). These data suggest that dysregulation of both Snord116 and Peg3 are important and associated with the physiological maintenance of OX neurons. Indeed, we observed that both genes were significantly altered only in narcoleptic mice, in which OX neurons and their cotransmitters were ablated, but not in narcoleptic mice lacking only Ppox.

Finally, to test whether the alteration of $P e g 3$ observed in the $\mathrm{PWScr}^{\mathrm{m}+\mathrm{p}-}$ mice and in the Atx mice is linked to the absence of Snord116 and whether Snord116 modulates the expression of Peg3, we used an RNA-interfering approach aimed at silencing Snord116 in immortalized embryonic rat hypothalamus cells (embryonic rat hypothalamus cell line R7). Specifically, cells transfected with a Snord116-specific siRNA showed significant Snord116 inhibition of approximately $80 \%$ at 48 hours after transfection (Figure 4C). However, the levels of Peg3 were unchanged (Figure 4C), indicating that Snord116 does not directly regulate the expression of the Peg3 gene. Pmch and Snurp were also unchanged (Supplemental Figure 5).

\section{Discussion}

Overall, this study shows evidence suggesting that Snord116 plays a crucial role in the regulation of REM sleep and thermoregulation, both phenotypes being dysregulated in $\mathrm{PWScr}^{\mathrm{m}+/ \mathrm{p}-}$ mutant mice 
A In vivo
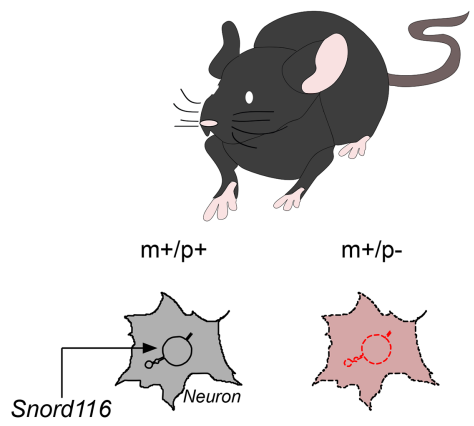

Peg3/Gapdh

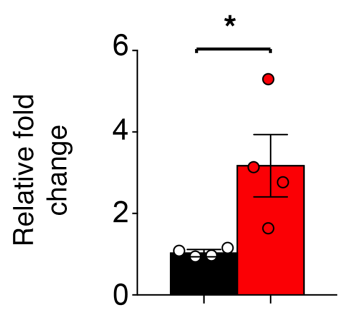

ChIP

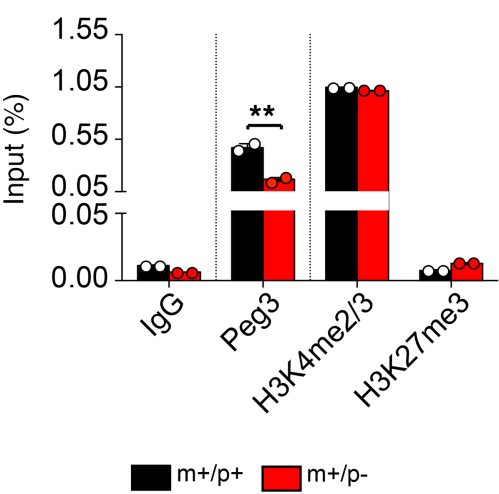

B In vivo
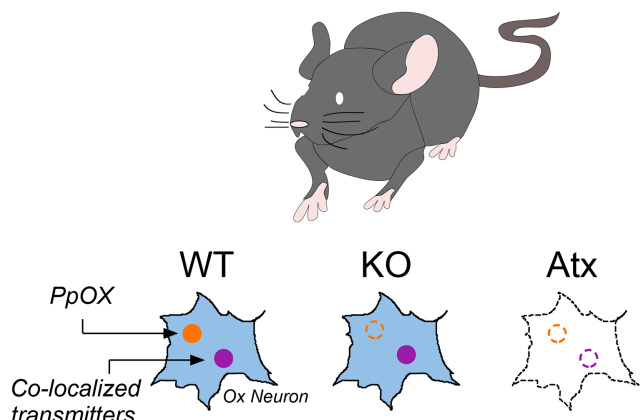

transmitters
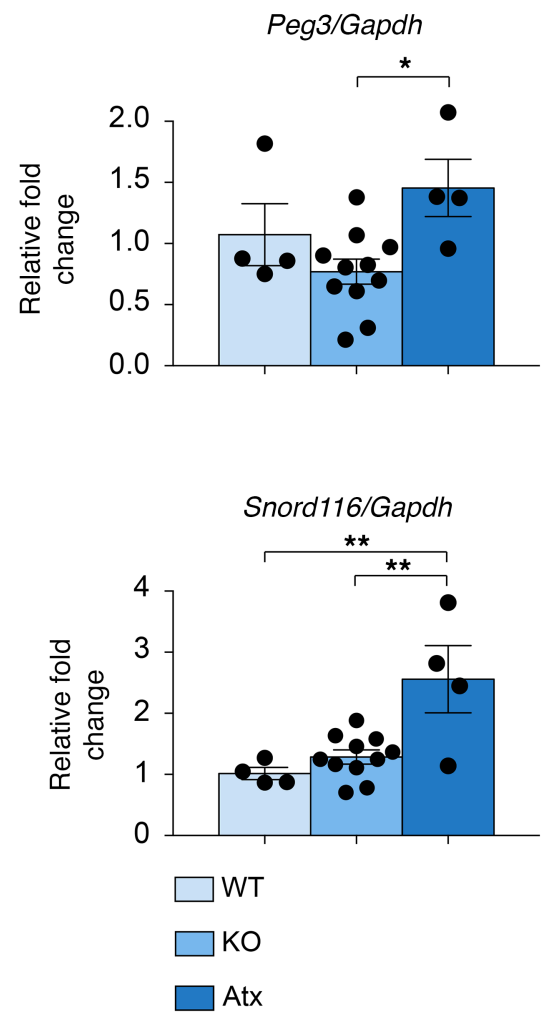

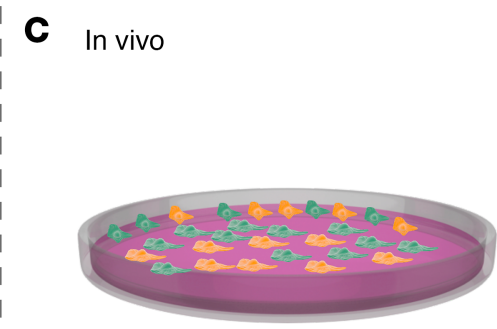

Embryonic rat hypothalamus cell line R7

Peg3/Gapdh

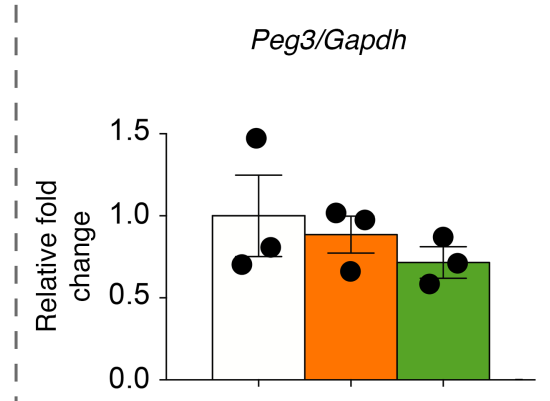

Snord116/Gapdh

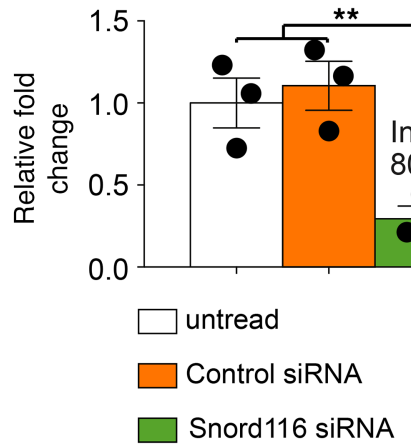

Figure 4. Snord116 and Peg3 play roles in the formation and maintenance of OX neurons. Peg3 regulates orexin expression in an independent manner from paternal Snord116. (A) Upper panel, the gene expression analysis of Peg3 in PWScr ${ }^{\mathrm{m}+/ \mathrm{p}-}$ mice (red) versus controls (black). Peg3 mRNA assessed

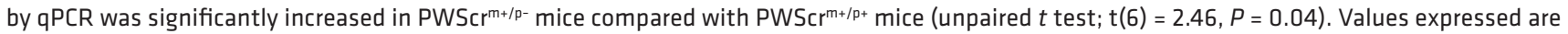
relative to the WT control mean \pm SEM. Gapdh was used as a housekeeping gene; see Supplemental Methods. Bottom panel, ChIP analysis of PEG3 binding to the Ppox promoter region in PWScr ${ }^{\mathrm{m}+/ \mathrm{p}-}$ mice (red) versus controls (black). PEC3 binding was lower in PWScr ${ }^{\mathrm{m}+/ \mathrm{p}^{-}}$mice than in PWScr ${ }^{\mathrm{m}+/ \mathrm{p}^{+}}$mice (unpaired $t$ test; $\mathrm{t}(2)=7.11, P=0.01$ ); see Supplemental Methods. (B) Peg3 gene expression (upper panel) and Snord116 gene expression (bottom panel) in Ppox-KO and orexin neuron-ablated (ataxin-3 [Atx] mice). One-way ANOVA indicated that Peg3 was significantly increased in Atx mice relative to KO mice, $\left(F_{[2,16]}=0.02\right.$, Bonferroni's post hoc test, $\left.P=0.03\right)$. Snord116 was increased in Atx mice relative to KO and control mice (WT) (1-way ANOVA; $\mathrm{F}_{[2,16]}=3.50$, Bonferroni's post hoc test, $\left.P=0.002\right)$. The following genotypes of narcoleptic mice were investigated: WT $(n=4), \mathrm{KO}(n=12)$, and Atx $(n=$ 4). (C) Snord116 and Peg3 gene expression analysis in the Snord116 siRNA-treated immortalized hypothalamic rat cell line. Snord116 siRNA (green bars) reduced the expression of the Snord116 gene compared with untreated cells or scrambled siRNA-treated cells (white and orange bars, respectively) (1-way ANOVA; $F_{[2,6]}=11.36$, Bonferroni's post hoc test, $P=0.009$ ). Peg3 mRNA levels were unchanged, similar to Snord116 siRNA, in untreated cells and scrambled siRNA-treated cells. The experiment was conducted in triplicate. Data are presented as the mean \pm SEM. ${ }^{*} P \leq 0.05 ;{ }^{*} P \leq 0.01$.

and patients $(5,38)$. The mouse model we studied here, however, also carries a partial deletion of the imprinted Ipw gene in PWS, which may contribute to the effects we reported. In particular, we observed REM sleep alterations at a particular time of the day when the pressure of sleep increases $(25,26)$; in mice, this occurs at a time in the sleep-wake cycle that corresponds to the "siesta time" 
$(25,26)$. This phenomenon has the potential to help future experimental designs aimed at addressing temporally precise therapy.

Snord116 is one of the most important candidate genes involved in the etiology of the major endophenotypes of PWS. Individuals lacking the SNORD116 small nucleolar RNA (snoRNA) cluster and the IPW gene suffer the same characteristics of hypotonia, hyperphagia, and failure to thrive that are observed in subjects with larger deletions and maternal uniparental disomy $(39,40)$. However, the Snord116 mouse model recapitulates all the sleep deficits of human PWS, but it only incompletely mimics the metabolic alterations. Indeed, both PWS mice and human patients show an alteration of body weight, but the 2 species show opposite phenotypes; indeed, PWS patients exhibit increased body weight, while mouse models across different studies show reduced weight $(21,41)$, displaying at least only the first stage (Stage I) of the PWS clinical features: the infancy stage (42). This overall result suggests that evolutionary divergence between the 2 species may have played an important role in the metabolism of PWS, but to date, there is no evidence for such a change.

We report for the first time to our knowledge an effect of the LH in Snord116 mutant mice, which is accompanied by an imbalance between $\mathrm{OX}$ and $\mathrm{MCH}$ neurons, causing a $60 \%$ reduction in OX neurons in the $\mathrm{LH}$, while $\mathrm{MCH}$ neurons were unaffected. Within the same 15q11-q13 region, it was previously reported that Magel2-KO mice lose $40 \%$ of OX neurons (28). These results suggest that multiple paternally expressed genes within the PWS region regulate the OX system in the LH, most likely in a dose-dependent manner. At BL, we observed that fire discharge of neurons associated with sleep (S-max neurons) are higher in mutant mice than in controls, suggesting that the role of Snord116 that originates from the LH is important in the determination of abnormal sleep in PWS. Indeed, several lines of evidence indicate that LH exerts a pivotal role in controlling the transition between sleep and wakefulness (15). Furthermore, while we observed that the proportion of S-max and W-max neurons significantly changed after 6 hours of SD with an increase in sleep neurons of approximately 13\% in WT control mice, PWS mutant mice lack this neuronal response by the LH to sleep loss. In the $\mathrm{LH}, \mathrm{OX}$ and $\mathrm{MCH}$ systems exert opposing effects on REM sleep (43); OX suppresses, while MCH promotes REM sleep. Therefore, the imbalance between OX and $\mathrm{MCH}$ systems in PWS mutant mice is in agreement with an increase in REM sleep.

Additionally, OX and MCH neurons in the LH regulate food intake and metabolism $(44,45)$. We found that mutant mice displayed a higher proportion of neurons - which were classified as nonresponding neurons (Type III) - than controls. In contrast, Type II neurons, which are downregulated in the LH during eating, were significantly reduced in mutant mice. It has been demonstrated that OX neurons decrease their firing during eating and remain in a depressed state throughout the entire eating phase (46). These findings suggest a lack of regulatory feedback mechanisms mediated by the OX system in relation to food intake in the LH of PWS model mice, a phenomenon that can relate to the hyperphagia and obesity phenotype observed in PWS patients. This evidence of neuromodulatory dysregulation of the LH is in agreement with previous results (20), which describe that a late onset of mild hyperphagia and obesity in mice can be induced only when Snord116 is selectively disrupted in the hypothalamus in adult mice.

Dysregulation of the OX system has been reported in a few clinical studies, although to date, the results remain contradictory. One study described an increase in the Orexin-A (OX-A) level in PWS subjects (47), while another showed a decrease in the peptide in the cerebrospinal fluid (11). Taken together, these findings call for an alteration of the OX system in PWS, although the contrasting results may also suggest that the loss of function of paternal alleles within the PWS region results in an increased phenotypic variance.

Our data suggest also that Snord116 is involved in the regulation, formation, and maintenance of the OX system in the LH. We assessed Snord116 expression in 2 different strains with OX deficiency, OX/Atx mice and OX peptide-KO mice $(9,36)$, and we found that only Atx mice showed a significant increase in Snord116. This line selectively loses OX neurons and their cotransmitters. In contrast, in KO mice that do not display a loss of OX neurons but are lacking OX peptides, the level of Snord116 was unaffected. The increase in Snord116 in mice with depletion of OX neurons is probably a compensatory mechanism. These results also suggest the role of Snord116, a paternally imprinted gene in regulating the hypothalamus and the OX system. Our assumption is also supported by a recent study (48) that describes the role of Peg3 in the development and expression of $\mathrm{OX}$ and $\mathrm{MCH}$ neurons. Our study indicates that PEG3 is able to bind OX promoters by increasing the expression of OX. However, a reduced binding of PEG3 was observed in mutant mice relative to control mice, which may be explained by a reduction in OX neurons. Thus, Peg3 was selectively altered in our $\mathrm{PWScr}^{\mathrm{m+/p-}}$ mutant mice, as well as 
in narcoleptic mice. In particular, Peg3 was found to be altered only in Atx narcoleptic mice, while it was unchanged in Ppox-KO mice. This may be explained by the fact that PEG3 binds the OX promoter, which is not altered in $\mathrm{KO}$ mice because they have a null mutation. Our study suggests that these 2 paternally imprinted genes, Snord116 and Peg3, are unlikely to interact with each other; however, both contribute to the development and OX neurons. Indeed, in our in vitro experiment with immortalized hypothalamic cells, knockdown of Snord116 did not directly change the levels of Peg3. This evidence is in line with the pioneering evidence in androgenetic mice (1) that paternal genome contains important contributions for the formation of the hypothalamus. In particular, our study implies that both Snord116 and Peg3 play a crucial role toward the formation of OX neurons. Therefore, this study advocates also for polygenic effect, the mechanism where multiple genes (imprinted genes) exert effects on the same trait, perhaps across different conditions.

We observed that $\mathrm{PWScr}^{\mathrm{m}+\mathrm{p}-}$ mutant mice present a high body temperature, coupled with an increase and lack of appropriate thermoregulatory responses. Thermoregulation is tightly integrated with the regulation of sleep and is also controlled by OX neuromodulation $(37,49)$. For example, narcoleptic subjects exhibit a paradoxically lower core body temperature while awake (50) and a higher body temperature during sleep $(51,52)$. In mammals, in physiological conditions, peripheral vasodilatation helps decrease the core body temperature during sleep initiation. Our mutant mice displayed a high body temperature during the light period, which corresponded to their resting phase/subjective sleep. Interestingly, narcoleptic mice show similar body temperature abnormalities (53). Moreover, when PWS mutant mice were kept at the TNZ, where resting metabolic rate remains stable and where REM sleep is preferentially increased (54, 55), we observed a surprising thermoregulatory response coupled with an altered homeostatic REM sleep response. REM sleep did not increase, and the peripheral thermoregulatory response of the mutant mice resembled what would be expected in a subneutrality $\left(22^{\circ} \mathrm{C}\right)$ environment. REM sleep is a stage of sleep in which thermoregulation is suspended; for this reason, REM sleep expression is more sensitive to ambient temperature manipulation than NREM sleep $(54,55)$. A recent study $(56)$ described that endotherms have evolved neural circuits to opportunistically promote REM sleep when the need for thermoregulatory defense is minimized, such as in TNZ conditions, suggesting a tight link between thermoregulation and REM sleep. In our study, the increase in body weight observed at the TNZ suggests that thermoneutrality is a permissive condition that induces body weight gain but does not compensate for the metabolic abnormality in these mice. Indeed, the differences with WT mice remained unchanged. Mutant mice showed growth retardation at both environmental conditions investigated - namely, at both $22^{\circ} \mathrm{C}$ and $30^{\circ} \mathrm{C}$.

The transcriptomic analysis suggests that loss of Snord116 might promote inflammatory responses and negatively affect synaptic organization in the hypothalamus, as previously observed in postmortem hypothalamic brain tissue from PWS patients (29). Additionally, we observed that SD impacts significantly the transcriptome of the hypothalamus in mutant mice compared with control mice, where only mild changes occur. Overall, the electrophysiological data together with the transcriptomic data suggest that loss of Snord116 leads to impaired homeostatic response that negatively affects the biological processes of the hypothalamus, and we observe that these effects are more pronounced in the context of SD. These data suggest that the sleep alterations observed in up to $76 \%$ of PWS patients $(57,58)$ represent a comorbidity that significantly affects the quality of life of these patients. Therefore, our results may provide compelling strategies to target dysregulated mechanisms in the hypothalamus of PWS patients affected by abnormal or poor sleep.

In conclusion, this evidence is in agreement with pioneer studies in androgenetic chimeric mice (1), where it was suggested that the paternal genome may account for regulatory mechanisms in the hypothalamus. Moreover, it reinforce the recent hypothesis that genomic imprinting plays a crucial role in mammalian sleep (3).

\section{Methods}

Animal husbandry, breeding, and genotyping

All animals were housed in controlled temperature conditions $\left(22^{\circ} \mathrm{C} \pm 1{ }^{\circ} \mathrm{C}\right)$ under a 12 -hour light/dark cycle (light on 8 a.m. -8 p.m.), with libitum access to food (standard chow diet) and water unless otherwise required by the experimental procedure.

Experiments were performed using adult mice at 15-17 weeks with paternal deletion of the Snord116 gene and $I P W$ exons A1/A2 exons (17) $\left(\mathrm{PWScr}^{\mathrm{m}+/ \mathrm{p}-}\right)$ and their WT littermates as control mice ( $\mathrm{PWScr}^{\mathrm{m}+/ \mathrm{p}^{+}}$). To maintain the colony, mice were bred and kept through paternal inheritance on a C57BL/6J background; 
mice were bred in the animal facility of Istituto Italiano di Tecnologia. Genotyping was performed as previously described (5). Briefly, PCR analysis of genomic DNA from ear punches was performed using the primer pair PWScrF1/PWScrR2 (5'-AGAATCGCTTGAACCCAGGA-3' and 5'-GAGAAGCCCTGTAACATGTCA-3', respectively). The deletion of PWScr resulted in a PCR product of approximately $300 \mathrm{bp}$, which was absent in the WT genotype.

For experiments in narcoleptic mice, 3 age-matched groups of male and female congenic mice from 2 different strains ( $\geq 9$ generations of backcrossing) on the C57BL/6J genetic background were used, as follows: (a) mice with congenital deficiency of OX gene (Ppox) KO (9) ( $n=11)$; (b) mice hemizygous for a transgene (Atx mice), the targeted expression of which causes selective ablation of OX neurons (36) $(n=4)$; and (c) WT controls $(n=4)$. Mouse colonies were maintained in the facilities of the Department of Biomedical and NeuroMotor Sciences at the University of Bologna, Italy. Mice were housed under a 12-hour light/ dark cycle at an ambient temperature of $25^{\circ} \mathrm{C}$ with free access to water and food (4RF21 diet, Mucedola).

All animal procedures were approved by the Animal Research Committee and the Veterinary Office of Italy for Istituto Italiano di Tecnologia. All efforts were made to minimize the number of animals used and any pain and discomfort according to the principles of the $3 \mathrm{Rs}(59)$.

\section{Experimental approach}

Neuronal activity in the mouse LH is time-locked with different behavioral and physiological states of the brain. To identify neuronal populations in the LH that control different physiological behaviors, we adopted a combined experimental approach (Figure 1A) in which we monitored single unit activity (SUA) at the LH in parallel with the cortical state of each animal by means of EEG/EMG recordings. The EEG/EMG information allows the identification of wakefulness, REM sleep, and NREM sleep states in the animal, which represent the 3 main behavioral states of the mammalian brain. Mice were kept throughout a regular sleep-wake cycle, and EEG/EMG/SUA were recorded during the light-to-dark transition (B1) and during the dark-to-light transition (B2) to test the neuronal responses during the minimum and maximum levels of sleep pressure, respectively (Figure 1B, upper panel). Then, each mouse underwent SD to test the homeostatic response following sleep loss (Figure 1B, middle panel; see Supplemental Methods for the SD procedures). Moreover, to test the other fundamental function of $\mathrm{LH}$, feeding, we food deprived each animal during the 12 hours of darkness of the light/dark cycle, and then, when food was made available, we evaluated the neuronal responses to food intake (Figure 1B, lower panel).

\section{Experimental protocols}

LH neuronal activity recordings. To investigate the neuronal dynamics of the LH, EEG/EMG and SUA were simultaneously recorded in 8 male adult $\mathrm{PWScr}^{\mathrm{m}+\mathrm{p}-}$ and $\mathrm{PWScr}^{\mathrm{m}+/ \mathrm{p}^{+}}$mice. Animals were individually housed after surgery in their home cages with a food hopper (a hole with an infrared beam) connected to a food dispenser that automatically delivered food pellets after a nose poke (powered by AM-Microsystems) (60). The food used was $20 \mathrm{mg}$ dustless precision pellets (Bioserv). After a 7-day recovery period after surgery, each mouse was connected to a flexible cable and swivel that allowed free movement within the cages; the mice were habituated for 2 days to the cable before SUA and EEG/EMG recordings (Figure 1A). Recordings of EEG/EMG with SUA were acquired at 2 different BL time points (B1 and B2; Figure 1A) for 2 hours according to the circadian process (C Process) and homeostatic process (S Process) of sleep. Next, to investigate sleep homeostasis, SUA and EEG/EMG were recorded during the first hour of the rebound period (RB, Figure 1A) following 6 hours of total SD and at other 2 time points over the 18-hour recovery period (SD1 and SD2, Figure $1 \mathrm{~A})$.

After a restoration period of 5 days from the previous $\mathrm{SD}$, animals were fasted for 12 hours during the dark period, which corresponds to the active phase of mice. At the beginning of the light period, mice were fed. A few food pellets were initially provided to encourage the mice to eat, and thereafter, food pellets were provided only after nose-poke activity. SUA was continuously recorded for 2 hours after being fed (Figure 1A). EEG/EMG was not recorded during this time. At the end of the last recording session, animals were sacrificed, and the locations of the recording electrodes were verified histologically (see the Supplemental Methods for the histology process).

Homeostatic investigation of sleep. To investigate the role of the Snord116 gene in the regulation of the homeostatic component of sleep, we recorded EEG/EMG in 10 male adult $\mathrm{PWScr}^{\mathrm{m}+\mathrm{p}-}$ and $\mathrm{PWScr}^{\mathrm{m}+/ \mathrm{p}^{+}}$ mice over 24 hours of $\mathrm{BL}$ at $22^{\circ} \mathrm{C} \pm 1^{\circ} \mathrm{C}$. The $\mathrm{BL}$ recordings began at $\mathrm{ZT} 0$ (the time of light onset in the 
12-hour light/dark cycle); then, mice were sleep deprived during the first 6 hours of the light phase (ZT $0-6)$ by gentle handling (introduction of novel objects into the cage, tapping on the cage, and when necessary, delicately touching) and then allowed 18 hours of recovery (ZT 6-24, RB).

Homeostatic investigation of REM sleep. To investigate the REM sleep propensity, we housed male adult $\mathrm{PWScr}^{\mathrm{m}+/ \mathrm{p}-}$ and $\mathrm{PWScr}{ }^{\mathrm{m}+\mathrm{p}+}$ mice for 5 weeks at $30^{\circ} \mathrm{C} \pm 1^{\circ} \mathrm{C}$, which is close to or within the TNZ of mice $(61,62)$. Then, these mice underwent continuous recordings for 24 hours at $30^{\circ} \mathrm{C}$ ambient temperature while undisturbed and freely moving in their cages.

\section{RNA-seq and analysis}

For this study, the whole hypothalamus of both genotypes of mice were investigated: $\mathrm{PWScr} \mathrm{m}^{\mathrm{t} / \mathrm{p}^{-}}$mice and $\mathrm{PWScr}^{\mathrm{m}+\mathrm{p}+}$ mice at 3 different time points at ZT 0 (G1; PWS, $n=3$, and WT, $n=3$ ), immediately after 6 hours of total SD (G2; PWS, $n=4$, and WT, $n=4$ ), and 1 hour after previous SD (G3; PWS, $n=4$, and WT, $n=4$ ). Total RNA was homogenized in Trizol Reagent (MilliporeSigma). Libraries were prepared using a TruSeq polyA mRNA kit (Illumina) according to the manufacturer's instructions and sequenced by using the NovaSeq 6000 System (Illumina). Raw sequence reads were quality controlled through FASTQC (https://www.bioinformatics.babraham.ac.uk/projects/fastqc/) and trimmed using Trimmomatic (v0.38) (63). To quantify the transcript abundances, we used kallisto (v0.44.0) (64). Kallisto was also used to build an index from the mouse reference genome (EnsDb.Mmusculus.v79) with default parameters. To import and summarize transcript-level abundance of kallisto, the $\mathrm{R}$ package tximport was used. Differential expression was assessed using DSeq2 (65). For FDR correction, adjusted $P$ values less than 0.05 were selected, and only genes with $>2$-fold change were considered in the analyses. The sequencing data are deposited in GEO accession number GSE139524 (https://www.ncbi.nlm.nih.gov/).

GO analysis was performed using Metascape (66) (http://metascape.org/). The identified DEGs are listed in Supplemental Tables 8 and 9. To compare mice and human DEGs in snord116 versus WT and PWS versus healthy controls, respectively, we ran a homology analysis of the human DEGs toward the mouse DEGs (noncoding RNAs were excluded from the analysis) and performed hypergeometric test. Significance of overrepresentation of GO terms was calculated using the hypergeometric test, corrected for multiple testing with a Benjamini \& Hochberg FDR correction (49), and a significance cutoff of 0.05 was applied to the result.

\section{Electrophysiological data analysis}

Cortical EEG/EMG signals were recorded using Dataquest ART (Data Science International). Signals were digitized at a sampling rate of $500 \mathrm{~Hz}$ with a filter cutoff of $50 \mathrm{~Hz}$. EEG signals were filtered at 0.3 $\mathrm{Hz}$ (low-pass filter) and $0.1 \mathrm{kHz}$ (high-pass filter). The polysomnographic recordings were visually scored offline using SleepSign software (Kissei Comtec Co. Ltd.) per 4-second epoch window to identify wakefulness, NREM, or REM sleep stages, as previously described $(67,68)$. Scoring was performed by a single observer who was blinded to the mouse groups.

Specifically, wakefulness, NREM, and REM states were scored when characteristic EEG/EMG activity occupied $75 \%$ of the epoch $(67,68)$. EEG epochs determined to have artefact (interference caused by scratching, movement, eating, or drinking) were excluded from the analysis. Artefact comprised $<5 \%-8 \%$ of all recordings used for analysis.

The percentage of time spent in total sleep, NREM, and REM sleep out of the total recording time was determined. The amount of time spent in each stage was established by counting the types of epochs (wakefulness, NR, or R) and averaging over 2-hour periods. The spectral characteristics of the EEG were further analyzed. The EEG power densities of the delta $(0,5-4 \mathrm{~Hz})$ and theta $(5-9 \mathrm{~Hz})$ frequencies in NREM and REM sleep were computed for all conditions investigated. The theta and delta power of each subject was normalized by the mean of the last 4 hours of the light period. To exclude variability due to the implantation, the power density of each animal was normalized to the power density of the last 4 hours of the light period of the 24 -hour recording $\left(22^{\circ} \mathrm{C}\right.$ and $30^{\circ} \mathrm{C}$, respectively). The temperature was recorded at a sampling rate of $5 \mathrm{~Hz}$ within the range of $34^{\circ} \mathrm{C}-41^{\circ} \mathrm{C}$ and averaged over 2-hour periods. A 2-way ANOVA with repeated measures (factors of group $\times$ time) was used for the statistical analysis of the 2-hour averaged time-course changes in the percentage of each sleep stage (wakefulness, NREM, and REM sleep), delta power, theta power, and body temperature between the 2 genotypes $\left({ }^{+/+}\right.$vs. $\left.^{-/}\right)$. The statistical analysis of the cumulative amount of wakefulness, NREM, and REM sleep over the dark and light periods among the groups at $\mathrm{BL}$, after $\mathrm{SD}$, and at the TNZ was performed with 1-way ANOVA. 
A 2-tailed unpaired $t$ test was used to compare differences in the sleep stages and in the delta and theta power during the first 4 hours of rebound after SD between the 2 genotypes.

SUA data acquisition was performed using an RX7 system (Tucker Davis Technology; TDT). The synchronization between the behavioral set up and the TDT system was guaranteed with transistor-transistor logic (TTL) from the CHORA feeders to the TDT system. The data were digitally sampled at $12 \mathrm{kHz}$. To detect spike timestamps, the neural traces were filtered with a bandpass filter $(300-5000 \mathrm{~Hz})$, and then, the common average reference (CAR) was applied (69). Spikes were detected using a hard threshold that was computed as previously described (70). A correlation filter was applied between each detected spike and the corresponding signal chunk in the other recording sites to detect and exclude false-positive spikes given by movement artefact.

To specifically identify active neurons in the LH, we imposed 2 criteria: (a) the refractory period following a spike was set to $1 \mathrm{~ms}$, and (b) the maximum duration of a waveform was set to $5 \mathrm{~ms}$. Spikes of individual neurons were sorted offline using the noise robust LDA-GMM algorithm based on a linear discriminant analysis (71). To identify neurons that specifically respond to a particular sleep-wake cycle, the unit activity was subsequently analyzed per 4-second epoch in each sleep-wake stage for the average discharge rate (spikes per second). Classification of units according to the state in which their maximal discharge rate occurred was performed by 1-way ANOVA followed by post hoc paired $t$ tests with the Bonferroni's correction, $(P<0.05)(72)$. This analysis allowed the classification of units into 3 main neuronal populations that were shown to respond during specific sleep-wake stages (Figure 1B).

Moreover, to identify the neurons related to feeding behavior in the $\mathrm{LH}$, we assessed the firing rate of neurons that responded after the food was released by aligning the unit firing to the pellet release from the CHORA feeder after a spontaneous nose-poke activity. The mean BL firing rate of each neuron was determined in the interval of 5 seconds before the pellet was released. Paired Student's $t$ tests were used to classify the firing rate differences of the same units before and after the food was released. To motivate the mice to perform more trials, they were food deprived for 12 hours during the dark period, and the recording session started at the beginning of the light period for the following 2 hours. Sessions were videotaped and reviewed to eliminate trials in which the mice performed the nose-poke activity but the food was not eaten. Video analysis also demonstrated that grooming rarely occurred and, thus, did not affect SUA. Indeed, grooming has been shown to induce moderate OX neuron activity (73). Among all sessions, a mean of $3.25 \pm 1.49$ trials for the $\mathrm{PWScr} \mathrm{m}^{\mathrm{m}+\mathrm{p}-}$ mice and 2 \pm 0.69 trials for the $\mathrm{PWScr}^{\mathrm{m}+/ \mathrm{p}-}$ mice were removed from the analysis because the food was not eaten.

\section{Statistics}

Values were tested for a Gaussian distribution with the Kolmogorov-Smirnov test. Data are presented as the mean \pm SEM. Two-way repeated-measures ANOVA was used to perform group comparisons with multiple measurements. Paired and unpaired $t$ tests were used for single value comparisons. One-way ANOVA was used to compare more than 2 groups, followed by post hoc Tukey's test. The Bonferroni's correction was further applied in the post hoc analysis, as appropriate, to correct for multiple comparisons. Neuronal dynamics of the LH were analyzed by the $\chi^{2}$ test. Phenopy (60) was used for sleep, temperature, and SUA analyses, while GraphPad Prism 6 was used for statistical analysis. Type I error $\alpha$ was set to $0.05(P<0.05)$.

\section{Study approval}

The study was approved by the ethics committee of the Italian Ministry of Healthy and subsequently approved by the ethics committee of the Istituto Italiano di Tecnologia (Genova, Italy; n.089).

\section{Author contributions}

MP designed the study. MP and MF performed the animal experiments. VT provided infrastructural support. $\mathrm{AF}$ and MP performed and analyzed the gene expression analysis. MP, EB, and MF performed the EEG and SUA analyses. AF performed the ChIP experiment. MC and RA analyzed and gave an interpretation of data from the infrared thermocamera. AU and FK analyzed the RNA-seq data. EH contributed resources. CB, VLM, and GZ provided and dissected brain samples from narcoleptic mice. MP, MF, and VT drafted and finalized the manuscript. All authors revised and finalized the manuscript.

\section{Acknowledgments}

We thank Simone Bellini, a master student of Tucci's laboratory, for her help with the RNA extraction and qPCR. This study was financially supported by Jerome Lejeune Foundation under grant agreements 
no. 1599-TV2016B and by the The Foundation for Prader Willi Research; this project received the Seal of Excellence by the European Union's Horizon 2020, N 753417. AU was financially supported by the Norwegian Cancer Society (grant no. 198016-2018).

Address correspondence to: Valter Tucci, Neurobehavioural Genetics Group, NBT - IIT Via Morego 30, 16163, Genova, Italy. Phone: 39.010.2896.747; Email: valter.tucci@iit.it.

1. Keverne EB, Fundele R, Narasimha M, Barton SC, Surani MA. Genomic imprinting and the differential roles of parental genomes in brain development. Brain Res Dev Brain Res. 1996;92(1):91-100.

2. Bonnavion P, Mickelsen LE, Fujita A, de Lecea L, Jackson AC. Hubs and spokes of the lateral hypothalamus: cell types, circuits and behaviour. J Physiol (Lond). 2016;594(22):6443-6462.

3. Tucci V. Genomic Imprinting: A New Epigenetic Perspective of Sleep Regulation. PLoS Genet. 2016;12(5):e1006004.

4. Tucci V, Isles AR, Kelsey G, Ferguson-Smith AC, Erice Imprinting Group. Genomic Imprinting and Physiological Processes in Mammals. Cell. 2019;176(5):952-965.

5. Lassi G, et al. Deletion of the Snord116/SNORD116 Alters Sleep in Mice and Patients with Prader-Willi Syndrome. Sleep. 2016;39(3):637-644

6. Holm VA, et al. Prader-Willi syndrome: consensus diagnostic criteria. Pediatrics. 1993;91(2):398-402.

7. Swaab DF. Prader-Willi syndrome and the hypothalamus. Acta Paediatr Suppl. 1997;423:50-54.

8. Vgontzas AN, et al. Daytime sleepiness and REM abnormalities in Prader-Willi syndrome: evidence of generalized hypoarousal. Int J Neurosci. 1996;87(3-4):127-139.

9. Chemelli RM, et al. Narcolepsy in orexin knockout mice: molecular genetics of sleep regulation. Cell. 1999;98(4):437-451

10. Mignot E, et al. The role of cerebrospinal fluid hypocretin measurement in the diagnosis of narcolepsy and other hypersomnias. Arch Neurol. 2002;59(10):1553-1562.

11. Omokawa M, et al. Decline of CSF orexin (hypocretin) levels in Prader-Willi syndrome. Am J Med Genet A. 2016;170A(5):1181-1186

12. Nevsimalova S, Vankova J, Stepanova I, Seemanova E, Mignot E, Nishino S. Hypocretin deficiency in Prader-Willi syndrome. Eur J Neurol. 2005;12(1):70-72.

13. Adamantidis AR, Zhang F, Aravanis AM, Deisseroth K, de Lecea L. Neural substrates of awakening probed with optogenetic control of hypocretin neurons. Nature. 2007;450(7168):420-424.

14. Schöne C, Burdakov D. Orexin/Hypocretin and Organizing Principles for a Diversity of Wake-Promoting Neurons in the Brain. Curr Top Behav Neurosci. 2017;33:51-74.

15. Jego S, et al. Optogenetic identification of a rapid eye movement sleep modulatory circuit in the hypothalamus. Nat Neurosci. 2013;16(11):1637-1643.

16. Seifinejad A, et al. Molecular codes and in vitro generation of hypocretin and melanin concentrating hormone neurons. Proc Natl Acad Sci USA. 2019;116(34):17061-17070.

17. Skryabin BV, et al. Deletion of the MBII-85 snoRNA gene cluster in mice results in postnatal growth retardation. PLoS Genet. 2007;3(12):e235.

18. Schoonakker M, Meijer JH, Deboer T, Fifel K. Heterogeneity in the circadian and homeostatic modulation of multiunit activity in the lateral hypothalamus. Sleep. 2018;41(6).

19. Hassani OK, Lee MG, Jones BE. Melanin-concentrating hormone neurons discharge in a reciprocal manner to orexin neurons across the sleep-wake cycle. Proc Natl Acad Sci USA. 2009;106(7):2418-2422.

20. Polex-Wolf J, et al. Hypothalamic loss of Snord116 recapitulates the hyperphagia of Prader-Willi syndrome. J Clin Invest. 2018;128(3):960-969.

21. Qi Y, et al. Snord116 is critical in the regulation of food intake and body weight. Sci Rep. 2016;6:18614.

22. Amici R, Zamboni G, Perez E, Jones CA, Parmeggiani PL. The influence of a heavy thermal load on REM sleep in the rat. Brain Res. 1998; 781(1-2):252-258.

23. Amici R, et al. Cold exposure and sleep in the rat: REM sleep homeostasis and body size. Sleep. 2008;31(5):708-715.

24. Szymusiak R, Satinoff E. Maximal REM sleep time defines a narrower thermoneutral zone than does minimal metabolic rate. Physiol Behav. 1981;26(4):687-690.

25. Ehlen JC, et al. Maternal Ube3a Loss Disrupts Sleep Homeostasis But Leaves Circadian Rhythmicity Largely Intact. J Neurosci. 2015;35(40):13587-13598.

26. Huber R, Deboer T, Tobler I. Effects of sleep deprivation on sleep and sleep EEG in three mouse strains: empirical data and simulations. Brain Res. 2000;857(1-2):8-19.

27. Li P, Sur SH, Mistlberger RE, Morris M. Circadian blood pressure and heart rate rhythms in mice. Am J Physiol. 1999;276(2):R500-R504.

28. Kozlov SV, et al. The imprinted gene Magel2 regulates normal circadian output. Nat Genet. 2007;39(10):1266-1272.

29. Bochukova EG, et al. A Transcriptomic Signature of the Hypothalamic Response to Fasting and BDNF Deficiency in Prader-Willi Syndrome. Cell Rep. 2018;22(13):3401-3408.

30. Zhou Y, et al. Metascape provides a biologist-oriented resource for the analysis of systems-level datasets. Nat Commun. 2019;10(1):1523.

31. Thiaville MM, Huang JM, Kim H, Ekram MB, Roh TY, Kim J. DNA-binding motif and target genes of the imprinted transcription factor PEG3. Gene. 2013;512(2):314-320.

32. Kuroiwa Y, et al. Peg3 imprinted gene on proximal chromosome 7 encodes for a zinc finger protein. Nat Genet. 1996;12(2):186-190.

33. Moriguchi T, Sakurai T, Takahashi S, Goto K, Yamamoto M. The human prepro-orexin gene regulatory region that activates gene expression in the lateral region and represses it in the medial regions of the hypothalamus. J Biol Chem. 
2002;277(19):16985-16992.

34. Ye A, He H, Kim J. PEG3 binds to H19-ICR as a transcriptional repressor. Epigenetics. 2016;11(12):889-900.

35. Bogliotti YS, Ross PJ. Mechanisms of histone H3 lysine 27 trimethylation remodeling during early mammalian development. Epigenetics. 2012;7(9):976-981.

36. Hara J, et al. Genetic ablation of orexin neurons in mice results in narcolepsy, hypophagia, and obesity. Neuron. 2001;30(2):345-354.

37. Kuwaki T. Thermoregulation under pressure: a role for orexin neurons. Temperature (Austin). 2015;2(3):379-391.

38. McVea S, Thompson AJ, Abid N, Richardson J. Thermal dysregulation in Prader-Willi syndrome: a potentially fatal complication in adolescence, not just in infancy. BMJ Case Rep. 2016;2016:bcr2016215344.

39. de Smith AJ, et al. A deletion of the HBII-85 class of small nucleolar RNAs (snoRNAs) is associated with hyperphagia, obesity and hypogonadism. Hum Mol Genet. 2009;18(17):3257-3265.

40. Sahoo T, et al. Prader-Willi phenotype caused by paternal deficiency for the HBII-85 C/D box small nucleolar RNA cluster. Nat Genet. 2008;40(6):719-721.

41. Ding F, et al. SnoRNA Snord116 (Pwcr1/MBII-85) deletion causes growth deficiency and hyperphagia in mice. PLoS One. 2008;3(3):e1709.

42. Miller JL, et al. Nutritional phases in Prader-Willi syndrome. Am J Med Genet A. 2011;155A(5):1040-1049.

43. Naganuma F, Bandaru SS, Absi G, Mahoney CE, Scammell TE, Vetrivelan R. Melanin-concentrating hormone neurons contribute to dysregulation of rapid eye movement sleep in narcolepsy. Neurobiol Dis. 2018;120:12-20.

44. Burdakov D, Karnani MM, Gonzalez A. Lateral hypothalamus as a sensor-regulator in respiratory and metabolic control. Physiol Behav. 2013;121:117-124.

45. Barson JR, Morganstern I, Leibowitz SF. Complementary roles of orexin and melanin-concentrating hormone in feeding behavior. Int J Endocrinol. 2013;2013:983964

46. González JA, Jensen LT, Iordanidou P, Strom M, Fugger L, Burdakov D. Inhibitory Interplay between Orexin Neurons and Eating. Curr Biol. 2016;26(18):2486-2491.

47. Manzardo AM, Johnson L, Miller JL, Driscoll DJ, Butler MG. Higher plasma orexin A levels in children with Prader-Willi syndrome compared with healthy unrelated sibling controls. Am J Med Genet A. 2016;170(8):2097-2102.

48. Seifinejad A, et al. Molecular codes and in vitro generation of hypocretin and melanin concentrating hormone neurons. Proc Natl Acad Sci USA. 2019;116(34):17061-17070.

49. Cerri M, et al. Enhanced slow-wave EEG activity and thermoregulatory impairment following the inhibition of the lateral hypothalamus in the rat. PLoS One. 2014;9(11):e112849.

50. Fronczek R, et al. Manipulation of core body and skin temperature improves vigilance and maintenance of wakefulness in narcolepsy. Sleep. 2008;31(2):233-240.

51. Mosko SS, Holowach JB, Sassin JF. The 24-hour rhythm of core temperature in narcolepsy. Sleep. 1983;6(2):137-146.

52. Pollak CP, Wagner DR. Core body temperature in narcoleptic and normal subjects living in temporal isolation. Pharmacol Biochem Behav. 1994;47(1):65-71.

53. Mochizuki T, Klerman EB, Sakurai T, Scammell TE. Elevated body temperature during sleep in orexin knockout mice. Am $J$ Physiol Regul Integr Comp Physiol. 2006;291(3):R533-R540.

54. Parmeggiani PL. Thermoregulation and sleep. Front Biosci. 2003;8:s557-s567.

55. Cerri M, Luppi M, Tupone D, Zamboni G, Amici R. REM Sleep and Endothermy: Potential Sites and Mechanism of a Reciprocal Interference. Front Physiol. 2017;8:624.

56. Komagata N, Latifi B, Rusterholz T, Bassetti CLA, Adamantidis A, Schmidt MH. Dynamic REM Sleep Modulation by Ambi ent Temperature and the Critical Role of the Melanin-Concentrating Hormone System. Curr Biol. 2019;29(12):1976-1987.e4.

57. Gunay-Aygun M, Schwartz S, Heeger S, O'Riordan MA, Cassidy SB. The changing purpose of Prader-Willi syndrome clinical diagnostic criteria and proposed revised criteria. Pediatrics. 2001;108(5):E92.

58. Williams K, Scheimann A, Sutton V, Hayslett E, Glaze DG. Sleepiness and sleep disordered breathing in Prader-Willi syndrome: relationship to genotype, growth hormone therapy, and body composition. J Clin Sleep Med. 2008;4(2):111-118.

59. Törnqvist E, Annas A, Granath B, Jalkesten E, Cotgreave I, Öberg M. Strategic focus on 3R principles reveals major reductions in the use of animals in pharmaceutical toxicity testing. PLoS ONE. 2014;9(7):e101638.

60. Balzani E, Falappa M, Balci F, Tucci V. An approach to monitoring home-cage behavior in mice that facilitates data sharing Nat Protoc. 2018;13(6):1331-1347.

61. Swoap SJ, Overton JM, Garber G. Effect of ambient temperature on cardiovascular parameters in rats and mice: a comparative approach. Am J Physiol Regul Integr Comp Physiol. 2004;287(2):R391-R396.

62. Speakman JR, Keijer J. Not so hot: Optimal housing temperatures for mice to mimic the thermal environment of humans. Mol Metab. 2012;2(1):5-9.

63. Bolger AM, Lohse M, Usadel B. Trimmomatic: a flexible trimmer for Illumina sequence data. Bioinformatics. 2014;30(15):2114-2120.

64. Bray NL, Pimentel H, Melsted P, Pachter L. Near-optimal probabilistic RNA-seq quantification. Nat Biotechnol. 2016;34(5):525-527

65. Love MI, Huber W, Anders S. Moderated estimation of fold change and dispersion for RNA-seq data with DESeq2. Genome Biol. 2014;15(12):550

66. Tripathi S, et al. Meta- and Orthogonal Integration of Influenza "OMICs" Data Defines a Role for UBR4 in Virus Budding Cell Host Microbe. 2015;18(6):723-735.

67. Pace M, Adamantidis A, Facchin L, Bassetti C. Role of REM Sleep, Melanin Concentrating Hormone and Orexin/Hypocretin Systems in the Sleep Deprivation Pre-Ischemia. PLoS One. 2017;12(1):e0168430.

68. Pace M, et al. Rapid eye movements sleep as a predictor of functional outcome after stroke: a translational study. Sleep. 2018;41(10).

69. Ludwig KA, Miriani RM, Langhals NB, Joseph MD, Anderson DJ, Kipke DR. Using a common average reference to improve cortical neuron recordings from microelectrode arrays. J Neurophysiol. 2009;101(3):1679-1689. 
70. Erickson JC, et al. Falling-edge, variable threshold (FEVT) method for the automated detection of gastric slow wave events in high-resolution serosal electrode recordings. Ann Biomed Eng. 2010;38(4):1511-1529.

71. Keshtkaran MR, Yang Z. Noise-robust unsupervised spike sorting based on discriminative subspace learning with outlier handling. J Neural Eng. 2017;14(3):036003.

72. Lee MG, Hassani OK, Jones BE. Discharge of identified orexin/hypocretin neurons across the sleep-waking cycle. J Neurosci. 2005;25(28):6716-6720.

73. Mileykovskiy BY, Kiyashchenko LI, Siegel JM. Behavioral correlates of activity in identified hypocretin/orexin neurons. Neuron. 2005;46(5):787-798. 